\title{
Exact three dimensional black hole with gauge fields in string theory
}

\author{
S. Hoseinzadeh ${ }^{\mathrm{a}}$, A. Rezaei-Aghdam ${ }^{\mathrm{b}}$ \\ Department of Physics, Faculty of Science, Azarbaijan Shahid Madani University, 53714-161 Tabriz, Iran
}

Received: 7 March 2015 / Accepted: 11 May 2015 / Published online: 27 May 2015

(C) The Author(s) 2015. This article is published with open access at Springerlink.com

\begin{abstract}
We have obtained exact three dimensional BTZ type solutions with gauge fields, for string theory on a gauge symmetric gravitational background constructed from semisimple extension of the Poincaré algebra (and the Maxwell algebra) in $2+1$ dimensions. We have studied the models for two non-Abelian and Abelian gauge fields solutions and shown that the related sigma model for each of these backgrounds is a $\mathrm{SL}(2, R)$ Wess-Zumino-Witten model and that these models are classically canonically equivalent. We have also obtained the dual solution for the Abelian case and by interpreting the new field strength tensors of the Abelian solution as electromagnetic field strength tensors shown that dual models coincide with the charged black string solution.
\end{abstract}

\section{Introduction}

In order to overcome some complexities of four dimensional gravity, many researchers have studied the gravity models in lower dimensions. For instance, they hope that some properties of lower dimensional black holes help them to model those of the four dimensional black holes. One of such attempts has resulted in the construction of the three dimensional rotating BTZ black hole $[1,2]$. Nearly two decades ago, Witten [3] has shown that an exact two dimensional black hole in string theory could be obtained by gauging a one dimensional subgroup $U(1)$ of $\operatorname{SL}(2, R)$. Also, exact three dimensional black string [4] and black hole [5] solutions in string theory have been obtained. In another attempt, the $\operatorname{SL}(2, R)$ WZW model and its relation to string theory in $A d S_{3}$ has been studied in detail in three different papers [6-8]. In those papers, the structure of the Hilbert space of the WZW model and the spectrum of physical states of the string theory have

\footnotetext{
a e-mail: hoseinzadeh@azaruniv.edu

b e-mail: rezaei-a@azaruniv.edu
}

been determined [6], and also the one-loop amplitude [7] and the correlation functions of the model [8] have been studied.

Recently, the Maxwell $[9,10]$ and semi-simple extension of Poincaré symmetries were applied to the construction of gauge symmetric gravity models in $3+1[11,12]$ and $2+1$ [13] dimensions (see also [14,15]). Here, we apply these symmetries to obtain an exact three dimensional black hole with gauge fields in string theory by introducing a new extended antisymmetric $B$-field and try to obtain some solutions for the equations of motion of the low energy string effective action $[16,17]$. The outline of the paper is as follows.

In Sect. 2, we construct a low energy string effective action in $2+1$ dimensions by use of new gauge field strengths and obtain its equations of motion. In Sect. 3, we solve the equations of motion using the BTZ metric and obtain two different non-Abelian and Abelian solutions (Abelian solution has no contribution of interaction terms in new gauge field strengths). Then we show that both solutions are exact solutions whereas the sigma models for each of these backgrounds is a $\operatorname{SL}(2, R)$ Wess-Zumino-Witten model. We also show that two sigma models corresponding to the two nonAbelian and Abelian solutions are classically canonically equivalent. We interpret the new gauge field strengths of the Abelian solution as an electromagnetic field strength tensors, and obtain the corresponding three different electric and magnetic fields. In Sect. 4, using duality transformation, we calculate dual solutions of the Abelian solution with respect to both spacelike $(\varphi)$ and timelike coordinate isometry symmetries and show that the dual solutions correspond to the charged black string solution. By obtaining both $\varphi$-dual and $t$-dual electric and magnetic fields, we show that duality relates the electric fields to the dual magnetic fields and vice versa. We present some concluding remarks in Sect. 5. In the appendix, we set both the Maxwell algebra and the semi-simple extension of the Poincare algebra (AdS- 
Lorentz algebra $\left.{ }^{1}\right)$ in $2+1$ dimensional spacetime based on the AdS algebra (so(2,2) in $2+1$ dimensions) by use of the S-expansion procedure with the appropriate semigroups $S$ and $\bar{S}[18,19]$.

\section{Gauge fields in string action from semi-simple extension of the Poincaré algebra in $2+1$ dimensions}

Let us consider the semi-simple extension of the Poincaré algebra (or the Maxwell algebra for $\lambda=0$ ) with the basis $X_{B}=\left\{P_{a}, J_{a}, Z_{a}\right\}$ in $2+1$ dimensions [13] as follows: ${ }^{2}$

$$
\begin{aligned}
& {\left[J_{a}, J_{b}\right]=\epsilon_{a b c} J^{c}, \quad\left[J_{a}, P_{b}\right]=\epsilon_{a b c} P^{c},} \\
& {\left[P_{a}, P_{b}\right]=k \epsilon_{a b c} Z^{c},} \\
& {\left[J_{a}, Z_{b}\right]=\epsilon_{a b c} Z^{c}, \quad\left[P_{a}, Z_{b}\right]=-\frac{\lambda}{k} \epsilon_{a b c} P^{c},} \\
& {\left[Z_{a}, Z_{b}\right]=-\frac{\lambda}{k} \epsilon_{a b c} Z^{c},}
\end{aligned}
$$

where $k$ and $\lambda$ are constants, and $Z_{a}$ are new generators which are added to ordinary Poincaré generators $P_{a}$ and $J_{a}$ to extend the algebra. One can construct gauge fields $h_{\mu}$ which are a Lie algebra valued one form $h=h_{\mu} \mathrm{d} x^{\mu}$ as follows:

$h_{\mu}(x)=h_{\mu}^{B}(x) X_{B}=e_{\mu}^{a}(x) P_{a}+\omega_{\mu}^{a}(x) J_{a}+A_{\mu}^{a}(x) Z_{a}$,

where $x^{\mu}(\mu=0,1,2)$ and $a, b=0,1,2$ are spacetime coordinates and Lie algebra indices, respectively. Using these gauge fields $\left(e_{\mu}^{a}(x), \omega_{\mu}^{a}(x), A_{\mu}^{a}(x)\right)$, one can write the new field strengths $F_{\mu \nu}^{a}$ as follows [13]:

$$
\begin{aligned}
F_{\mu \nu}^{a}= & \partial_{[\mu} A_{\nu]}^{a}+\epsilon_{b c}^{a}\left(k e_{\mu}^{b} e_{\nu}^{c}+\omega_{\mu}^{b} A_{\nu}^{c}\right. \\
& \left.+A_{\mu}^{b} \omega_{\nu}^{c}\right)-\frac{\lambda}{k} \epsilon_{b c}^{a} A_{\mu}^{b} A_{\nu}^{c},
\end{aligned}
$$

where the gauge fields $e_{\mu}^{a}, \omega_{\mu}^{a}$ and $A_{\mu}^{a}$ are vierbein, spin connection and new non-Abelian gauge fields ${ }^{3}$ (correspond-

1 The semi-simple extension of the Poincaré algebra is the direct sum of the anti-de Sitter (AdS) algebra and the Lorentz algebra, i.e. $s o(2,2) \oplus$ $\operatorname{so}(2,1)$ in $2+1$ dimensional spacetime, and then is called the AdSLorentz algebra.

2 The relationship between the Maxwell algebra and the semi-simple extension of the Poincare algebra with the AdS algebra is discussed in the appendix.

${ }^{3}$ Note that for the general form of the field strength (3) the gauge fields $A_{\mu}^{a}$ are non-Abelian. For the Abelian case we have

$F_{\mu \nu}^{a}=\partial_{\mu} A_{\nu}^{a}-\partial_{\nu} A_{\mu}^{a}$,

i.e. for these gauge fields $A_{\mu}^{a}$ we have

$\epsilon_{b c}^{a}\left(k e_{\mu}^{b} e_{v}^{c}+\omega_{\mu}^{b} A_{v}^{c}+A_{\mu}^{b} \omega_{v}^{c}\right)-\frac{\lambda}{k} \epsilon_{b c}^{a} A_{\mu}^{b} A_{v}^{c}=0$. ing to the new generators $Z_{a}$ ), respectively. Now we consider the low energy string effective action in $2+1$ dimensional spacetime $\mathcal{M}$ by use of these gauge fields as follows ${ }^{4}[5,16]$ :

$$
\begin{aligned}
S= & \int_{\mathcal{M}} \mathrm{d}^{3} x \sqrt{-g} \mathrm{e}^{-2 \phi}\left[R+\frac{4}{K}+4(\nabla \phi)^{2}-\frac{1}{12} H_{\mu \nu \rho} H^{\mu \nu \rho}\right. \\
& \left.-\frac{1}{12} H_{\mu \nu \rho}^{\prime a} H_{a}^{\prime \mu \nu \rho}\right]
\end{aligned}
$$

where $R$ is the Ricci scalar of $\mathcal{M}$ and $\phi$ is the dilaton field, and furthermore $H_{\mu \nu \rho}$ and $H_{\mu \nu \rho}^{\prime} a 5$ are defined as follows:

$H_{\mu \nu \rho}=\partial_{\mu} B_{\nu \rho}+\partial_{\nu} B_{\rho \mu}+\partial_{\rho} B_{\mu \nu}$,
$H_{\mu \nu \rho}^{\prime a}=\partial_{\mu} F_{\nu \rho}^{a}+\partial_{\nu} F_{\rho \mu}^{a}+\partial_{\rho} F_{\mu \nu}^{a}$,

so that $B_{\mu \nu}$ is an antisymmetric field and $F_{\mu \nu}{ }^{a}$ are the new antisymmetric field strengths. Now, one can find the following equations of motion (the beta functions) by variation of the above action with respect to $g^{\mu \nu}, B^{v \rho}, F_{a}^{v \rho}$, and $\phi$, respectively:

$$
\begin{aligned}
& R_{\mu \nu}+2 \nabla_{\mu} \nabla_{\nu} \phi-\frac{1}{4} H_{\mu \rho \sigma} H_{\nu}{ }^{\rho \sigma}-\frac{1}{8} H_{\mu \rho \sigma}^{\prime a} H_{\nu}^{\prime}{ }_{a}^{\rho \sigma}=0 \\
& \nabla^{\mu}\left(\mathrm{e}^{-2 \phi} H_{\mu \nu \rho}\right)=0 \\
& \nabla^{\mu}\left(\mathrm{e}^{-2 \phi} H_{\mu \nu \rho}^{\prime a}\right)=0 \\
& 4 \nabla^{2} \phi-4(\nabla \phi)^{2}+R+\frac{4}{K} \\
& \quad-\frac{1}{12} H_{\mu \nu \rho} H^{\mu \nu \rho}-\frac{1}{12} H_{\mu \nu \rho}^{\prime a} H_{a}^{\prime \mu \nu \rho}=0,
\end{aligned}
$$

where these equations are zeros of the beta functions (at one loop) $\beta(G), \beta(B), \beta\left(F^{a}\right)$, and $\beta(\phi)$ (respectively) for the following sigma model [17]:

$$
\begin{aligned}
I= & \int_{\Sigma} \mathrm{d}^{2} \sigma \sqrt{g}\left(G_{\mu \nu}(X) \partial_{\alpha} X^{\mu} \partial_{\beta} X^{\nu} g^{\alpha \beta}\right. \\
& \left.+B_{\mu \nu}^{\prime}(X) \partial_{\alpha} X^{\mu} \partial_{\beta} X^{\nu} \epsilon^{\alpha \beta}+\frac{1}{2} R^{(2)} \phi(X)\right),
\end{aligned}
$$

where $\Sigma$ is the worldsheet, $R^{(2)}$ is the scalar curvature of the worldsheet metric $g_{\alpha \beta}$, and $\epsilon^{\alpha \beta}$ is an antisymmetric 2tensor, normalized so that $\sqrt{g} \epsilon^{12}=+1$, and the extended antisymmetric $B$-field has the following form:

$$
\begin{aligned}
B_{\mu \nu}^{\prime}(X) & =B_{\mu \nu}(X)+F_{\mu \nu}^{0}(X)+F_{\mu \nu}^{1}(X)+F_{\mu \nu}^{2}(X) \\
& =B_{\mu \nu}(X)+F_{\mu \nu}^{a}(X) \xi_{a},
\end{aligned}
$$

so that we have $\xi_{a}=(1,1,1)$.

\footnotetext{
${ }^{4}$ Note that the above action is the ordinary string effective action in $2+1$ dimensions where the last term is added.

5 The algebra indices $a$ can be taken up and down by the ad-invariant metric $\Omega_{a b}$ of the algebra [13].
} 


\section{Black hole solutions}

In this section, we will try to obtain a solution for the equations (7). We know that the $2+1$ dimensional EinsteinHilbert action with cosmological constant term,

$S=\int \mathrm{d}^{3} x \sqrt{-g}(R-2 \Lambda)$,

has a BTZ black hole solution as follows [1,2]:

$\mathrm{d} s^{2}=-N^{2}(r) \mathrm{d} t^{2}+\frac{1}{N^{2}(r)} \mathrm{d} r^{2}+r^{2}\left(N^{\phi}(r) \mathrm{d} t+\mathrm{d} \phi\right)^{2}$,

with

$N^{2}(r)=-M+\frac{r^{2}}{\ell^{2}}+\frac{J^{2}}{4 r^{2}}, \quad N^{\phi}(r)=-\frac{J}{2 r^{2}}$,

where $\Lambda=-\frac{1}{\ell^{2}}$ is the negative cosmological constant, furthermore $M$ and $J$ are the mass and angular momentum of the black hole, respectively. Here $\left[x^{0}, x^{1}, x^{2}\right]=[t, r, \varphi]$ are the coordinates of the spacetime. We use the above BTZ metric to solve the equations of motion (7) assuming that all fields are a function of the radial coordinate only. Here, we analyze two interesting non-Abelian and Abelian gauge field solutions.

\subsection{Non-Abelian case}

Using the form of the metric in (11) and (12) the equations of motion (7) have a solution as follows:

$$
\begin{aligned}
B_{20}(r)= & \frac{r^{2}}{\ell}, \quad \phi(r)=0, \quad K=\ell^{2}, \\
\omega^{0}(r)= & \frac{v(r)}{D_{2} r^{2}+D_{1}}\left( \pm r^{2} \sqrt{D_{2}^{2}-D_{4}^{2}}-k r N(r)+D_{5}\right) \\
& \mathrm{d} t+u(r) \mathrm{d} r, \\
\omega^{1}(r)= & w(r) \mathrm{d} r, \quad \omega^{2}(r)=v(r) \mathrm{d} t+z(r) \mathrm{d} r, \\
A^{0}(r)= & \frac{D_{4} r^{2}+D_{3}}{v(r)}\left(-\frac{J}{2 r^{2}} \mathrm{~d} t+\mathrm{d} \varphi\right)+y(r) \mathrm{d} r, \\
A^{1}(r)= & \frac{D_{2} r^{2}+D_{1}}{v(r)}\left(-\frac{J}{2 r^{2}} \mathrm{~d} t+\mathrm{d} \varphi\right)+q(r) \mathrm{d} r, \\
& A^{2}(r)=s(r) \mathrm{d} r,
\end{aligned}
$$

where $D_{1}, D_{2}, D_{3}, D_{4}, D_{5}$ are arbitrary constants and $v(r) \neq$ $0, u(r), w(r), y(r), q(r), s(r), z(r)$ are arbitrary functions of the radial coordinate $r$ only. Now, using (3), we find the following nonzero components of the new antisymmetric fields (3) for the non-Abelian gauge fields $A_{\mu}^{a}$ :

$$
\begin{aligned}
& F_{20}{ }^{0}(r)=D_{2} r^{2}+D_{1}, \\
& F_{20}{ }^{1}(r)=D_{4} r^{2}+D_{3}, \\
& F_{20}{ }^{2}(r)=r^{2} \sqrt{D_{2}^{2}-D_{4}^{2}}+D_{5},
\end{aligned}
$$

which yields the following extended antisymmetric B-field:

$$
\begin{aligned}
& B_{20}^{\prime}(r)=B_{20}(r)+F_{20}{ }^{0}(r)+F_{20}^{1}(r)+F_{20}^{2}(r) \\
& =\left(\frac{1}{\ell}+D_{2}+D_{4}+\sqrt{D_{2}^{2}-D_{4}^{2}}\right) r^{2}+D_{1}+D_{3}+D_{5} .
\end{aligned}
$$

Although this is a solution for the one-loop beta function equations, one can show by selecting $D_{2}=D_{4}=-\frac{1}{\ell}$ that this solution is also an exact solution of the beta function equations in all loops. Indeed, the sigma model (8) with this background $\left(G_{\mu \nu}, B_{\mu \nu}^{\prime}\right)$ is a $\operatorname{SL}(2, R)$ Wess-Zumino-Witten model. One can easily check this, by using the following $\operatorname{SL}(2, R)$ group element:

$g(t, r, \varphi)=\left(\begin{array}{cc}\hat{r} \mathrm{e}^{-\hat{\varphi}} & \sqrt{\ell^{2}-\hat{r}^{2}} \mathrm{e}^{\hat{t}} \\ \sqrt{\ell^{2}-\hat{r}^{2}} \mathrm{e}^{-\frac{\hat{t}}{\ell}} & -\hat{r} \mathrm{e}^{\hat{\varphi}}\end{array}\right)$,

in the WZW action

$S_{\mathrm{WZW}}=\frac{\bar{k}}{4 \pi} \int \mathrm{d}^{2} z \operatorname{Tr}\left(g^{-1} \partial g g^{-1} \bar{\partial} g\right)-\frac{\bar{k}}{12 \pi} \int \operatorname{Tr}\left(g^{-1} \mathrm{~d} g\right)^{3}$,

where

$\hat{r}=\ell \sqrt{\frac{r^{2}-r_{-}^{2}}{r_{+}^{2}-r_{-}^{2}}}, \quad \hat{t}=\frac{r_{+}}{\ell} t+r_{-} \varphi, \quad \hat{\varphi}=\frac{r_{-}}{\ell^{2}} t+\frac{r_{+}}{\ell} \varphi$,

so that $\bar{k}$ is the level of the WZW model, and the horizons $r=r_{ \pm}$of the black hole have the following relation to its mass and angular momentum $(M, J)$ :

$r_{ \pm}=\frac{\ell}{2}\left(\sqrt{M+\frac{J}{\ell}} \pm \sqrt{M-\frac{J}{\ell}}\right)$

\subsection{Abelian (electromagnetic) case}

Using the form of the metric in (11) and (12), we find another solution for the equations of motion (7) as follows:

$$
\begin{aligned}
B_{20}(r)= & \frac{r^{2}}{\ell}, \quad \phi(r)=0, \quad K=\ell^{2}, \\
\omega^{0}(r)= & \frac{g(r)}{r} N(r) \mathrm{d} t \\
& +\left(\frac{-f(r) h(r) g(r)+p(r)\left(\lambda r^{2}+(g(r))^{2}\right)}{k r^{2}}\right) \mathrm{d} r, \\
\omega^{1}(r)= & -\frac{J}{2 r^{2}} g(r) \mathrm{d} t+f(r) \mathrm{d} r+g(r) \mathrm{d} \varphi, \\
\omega^{2}(r)= & \frac{-\lambda r}{g(r) N(r)} \mathrm{d} r, \\
A^{0}(r)= & -\frac{J}{2 r^{2}} h(r) \mathrm{d} t+p(r) \mathrm{d} r+h(r) \mathrm{d} \varphi, \\
A^{1}(r)= & \frac{J k}{2 g(r)} \mathrm{d} t-\frac{k r^{2}}{g(r)} \mathrm{d} \varphi, \quad A^{2}(r)=\frac{-k r}{g(r) N(r)} \mathrm{d} r,
\end{aligned}
$$


where $g(r) \neq 0, f(r), h(r), p(r)$ are arbitrary functions of the radial coordinate only. Note that for this solution $A_{\mu}^{a}$ is Abelian and hence yields zero contributions for the coupling terms in all components of the new field strengths $F_{\mu \nu}^{a}$ (i.e. we have $\epsilon_{b c}{ }^{a}\left(k e_{\mu}^{b} e_{\nu}^{c}+\omega_{\mu}^{b} A_{v}^{c}+A_{\mu}^{b} \omega_{\nu}^{c}\right)-\frac{\lambda}{k} \epsilon_{b c}{ }^{a} A_{\mu}^{b} A_{v}^{c}=$ 0 ), and therefore $F_{\mu \nu}{ }^{a}$ can be interpreted as electromagnetic field strength tensors without coupling terms as follows:

$F_{\mu \nu}^{a}=\partial_{\mu} A_{\nu}^{a}-\partial_{\nu} A_{\mu}^{a}$.

For the above solution, all of the nonzero components of the electromagnetic field strength tensors are as follows:

$F_{01}{ }^{0}(r)=-\frac{J}{r^{3}} h(r)+\frac{J}{2 r^{2}} \frac{\mathrm{d}}{\mathrm{d} r} h(r), F_{21}{ }^{0}(r)=-\frac{\mathrm{d}}{\mathrm{d} r} h(r)$,

$F_{01}^{1}(r)=\frac{J k}{2 g^{2}(r)} \frac{\mathrm{d}}{\mathrm{d} r} g(r), F_{21}^{1}(r)=\frac{2 k r}{g(r)}-\frac{k r^{2}}{g^{2}(r)} \frac{\mathrm{d}}{\mathrm{d} r} g(r)$.

In this way, the extended $B$-field components have the following forms:

$$
\begin{aligned}
B_{20}^{\prime}(r) & =B_{20}(r)=\frac{r^{2}}{\ell}, \\
B_{01}^{\prime}(r) & =B_{01}(r)+F_{01}^{0}(r)+F_{01}{ }^{1}(r)+F_{01}^{2}(r) \\
& =-\frac{J}{r^{3}} h(r)+\frac{J}{2 r^{2}} \frac{\mathrm{d}}{\mathrm{d} r} h(r)+\frac{J k}{2 g^{2}(r)} \frac{\mathrm{d}}{\mathrm{d} r} g(r), \\
B_{21}^{\prime}(r) & =B_{21}(r)+F_{21}{ }^{0}(r)+F_{21}{ }^{1}(r)+F_{21}{ }^{2}(r) \\
& =-\frac{\mathrm{d}}{\mathrm{d} r} h(r)+\frac{2 k r}{g(r)}-\frac{k r^{2}}{g^{2}(r)} \frac{\mathrm{d}}{\mathrm{d} r} g(r),
\end{aligned}
$$

where we assume that $B_{01}(r)=B_{21}(r)=0$. This solution is also an exact solution. One can show that using the $\operatorname{SL}(2, R)$ group element (16) with

$\hat{r}=\ell \sqrt{\frac{r^{2}-r_{-}^{2}}{r_{+}^{2}-r_{-}^{2}}}, \quad \hat{t}=\frac{r_{+}}{\ell} t-r_{-} \varphi, \quad \hat{\varphi}=\frac{r_{-}}{\ell^{2}} t-\frac{r_{+}}{\ell} \varphi$,

the sigma model (8) with the background $\left(G_{\mu \nu}, B_{\mu \nu}^{\prime}\right)$ is a $\operatorname{SL}(2, R) W Z W$ model. Now, we show that the two sigma models corresponding to the two discussed non-Abelian (13) and Abelian (19) solutions are classically canonically equivalent. Indeed, by assuming the following relations among the arbitrary functions in (13) and (19):

$$
\begin{aligned}
v(r)= & \frac{2 r^{2} \sqrt{-\Lambda}}{h(r)-\frac{k r^{2}}{g(r)}-\int \mathrm{d} r \frac{k r}{N(r)}+D}, \\
y(r)= & \left(\frac{J k}{r g(r)}-\frac{J h(r)}{r^{3}}\right) \frac{1}{v(r)}+\frac{2 J \sqrt{-\Lambda}}{r v^{2}(r)} \\
& -q(r)+\frac{k N(r)}{r \sqrt{-\Lambda}}(s(r)-q(r)), \\
u(r)= & w(r)-2 z(r)+\frac{\lambda}{k}(y(r)-q(r)+2 s(r)),
\end{aligned}
$$

these two sigma models can be canonically related to each other by the following relations [20]:

$$
\begin{aligned}
& \left(C^{-1}\right)^{\mu \rho}\left(G_{\rho \lambda}+B_{\rho \lambda}^{\prime}+A_{\rho \lambda}\right)\left(C^{-1}\right)^{\kappa \lambda}\left(\widetilde{G}_{\kappa \nu}+\widetilde{B}_{\kappa \nu}^{\prime}+\widetilde{A}_{\kappa \nu}\right) \\
& \quad=\delta^{\mu}{ }_{\nu},\left(G_{\mu \nu}+B_{\mu \nu}^{\prime}\right)=\left(G_{\mu \rho}+B_{\mu \rho}^{\prime}+A_{\mu \rho}\right) \\
& \left(C^{-1}\right)^{\lambda \rho}\left(\widetilde{G}_{\lambda \nu}+\widetilde{B}_{\lambda \nu}^{\prime}\right),
\end{aligned}
$$

where $\left(G_{\mu \nu}, B_{\mu \nu}^{\prime}\right)$ and $\left(\widetilde{G}_{\mu \nu}, \widetilde{B}_{\mu \nu}^{\prime}\right)$ are the non-Abelian (13) and the Abelian (19) solutions respectively, such that $C_{\mu \nu}, A_{\mu \nu}$ and $\widetilde{A}_{\mu \nu}$ have the following forms:

$C_{\mu \nu}=G_{\mu \nu}+B_{\mu \nu}^{\prime}, \quad A_{\mu \nu}=\widetilde{A}_{\mu \nu}=0$.

Now, for the Abelian solution, the electric and magnetic fields can be written in terms of the electromagnetic field strength tensors as follows:

$$
\begin{aligned}
E_{r}^{(a)} & =-F^{01 a}=-\left(g^{00} g^{11} F_{01}{ }^{a}+g^{02} g^{11} F_{21}{ }^{a}\right) \\
& =F_{01}{ }^{a}-N^{\phi}(r) F_{21}{ }^{a}, \quad E_{\varphi}^{(a)}=0, \\
B_{z}^{(a)} & =r F^{12 a}=-r\left(g^{11} g^{20} F_{01}{ }^{a}+g^{11} g^{22} F_{21}{ }^{a}\right) \\
& =\frac{J}{2 r} F_{01}{ }^{a}-\left(\frac{N^{2}-r^{2}\left(N^{\phi}\right)^{2}}{r}\right) F_{21}{ }^{a},
\end{aligned}
$$

where the indices inside the parentheses are algebra indices which run over $0,1,2$ and each denotes a different field. In this way, we have three different electric and magnetic fields. The radial components of three electric fields have the following forms:

$E_{r}^{(0)}=-\frac{J}{r^{3}} h(r), \quad E_{r}^{(1)}=\frac{J k}{r g(r)}, \quad E_{r}^{(2)}=0 ;$

furthermore, all of the azimuthal components of them are zero

$E_{\varphi}^{(0)}=E_{\varphi}^{(1)}=E_{\varphi}^{(2)}=0$,

and all of the magnetic fields are in the z-direction, i.e. they are perpendicular to the $r, \phi$ plane. These magnetic fields are given by

$$
\begin{aligned}
B_{z}^{(0)}= & -\frac{J^{2}}{2 r^{4}} h(r)+\frac{N^{2}}{r} \frac{\mathrm{d}}{\mathrm{d} r} h(r), \quad B_{z}^{(1)}=\frac{2 k\left(M+\Lambda r^{2}\right)}{g(r)} \\
& +\frac{k r N^{2}}{(g(r))^{2}} \frac{\mathrm{d}}{\mathrm{d} r} g(r), \quad B_{z}^{(2)}=0 .
\end{aligned}
$$

\section{Dual solution}

Now, we will try to find the dual of the Abelian solution (19), which is indeed another solution for the equations of motion. Note that the solution (19) is independent of the coordinate $\varphi$; hence we have an isometry in the $\varphi$-direction. Abelian duality, which corresponds to a transformation on the 
string model, relates this solution $\left(g_{\mu \nu}, B_{\mu \nu}, F_{\mu \nu}^{a}, \phi\right)$ to the dual solution $\left(\tilde{g}_{\mu \nu}, \tilde{B}_{\mu \nu}, \tilde{F}_{\mu \nu}^{a}, \tilde{\phi}\right)$ by the following Buscher transformation $[21,22]$ :

$$
\begin{aligned}
\tilde{g}_{22} & =\frac{1}{g_{22}}, \quad \tilde{g}_{\alpha \beta}=g_{\alpha \beta}-\frac{\left(g_{2 \alpha} g_{2 \beta}-B_{2 \alpha}^{\prime} B_{2 \beta}^{\prime}\right)}{g_{22}}, \\
\tilde{g}_{2 \alpha} & =\frac{B_{2 \alpha}^{\prime}}{g_{22}}, \quad \tilde{B}_{\alpha \beta}^{\prime}=B_{\alpha \beta}^{\prime}-\frac{2 g_{2[\alpha} B_{\beta] 2}^{\prime}}{g_{22}}, \\
\tilde{B}_{2 \alpha}^{\prime} & =\frac{g_{2 \alpha}}{g_{22}}, \quad \tilde{\phi}=\phi-\frac{1}{2} \ln \left(g_{22}\right),
\end{aligned}
$$

where $\alpha, \beta=0,1$ (the index ' 2 ' represents the $\varphi$ coordinate). Applying this transformation to the solution (11), (12), (19), and (21) yields the following dual solution:

$$
\begin{aligned}
& \tilde{\mathrm{d} s}^{2}=\left(M-\frac{J^{2}}{4 r^{2}}\right) \mathrm{d} t^{2}+\frac{2}{\ell} \mathrm{d} t \mathrm{~d} \varphi+2 B_{21}^{\prime}(r)\left(\frac{1}{\ell} \mathrm{d} t \mathrm{~d} r+\frac{1}{r^{2}} \mathrm{~d} r \mathrm{~d} \varphi\right) \\
& \quad+\frac{1}{r^{2}} \mathrm{~d} \varphi^{2}+\left\{\frac{1}{N^{2}(r)}+\frac{1}{r^{2}}\left(B_{21}^{\prime}(r)\right)^{2}\right\} \mathrm{d} r^{2}, \\
& \tilde{B}_{20}(r)=-\frac{J}{2 r^{2}}, \quad \tilde{\phi}(r)=-\ln (r), \\
& \tilde{F}_{01}{ }^{0}(r)=-\frac{J}{r^{3}} h(r)+\frac{3 J}{2 r^{2}} \frac{\mathrm{d}}{\mathrm{d} r} h(r), \\
& \tilde{F}_{01}{ }^{1}(r)=-\frac{2 J k}{r g(r)}+\frac{3 J k}{2 g^{2}(r)} \frac{\mathrm{d}}{\mathrm{d} r} g(r),
\end{aligned}
$$

where the dual electromagnetic field strengths $\tilde{F}_{01}{ }^{0}$ and $\tilde{F}_{01}{ }^{1}$ are related to the following gauge fields (using (20)):

$$
\begin{aligned}
& \tilde{A}_{0}^{0}(r)=-\int \mathrm{d} r\left(-\frac{J}{r^{3}} h(r)+\frac{3 J}{2 r^{2}} \frac{\mathrm{d}}{\mathrm{d} r} h(r)\right), \\
& \tilde{A}_{0}^{1}(r)=-\int \mathrm{d} r\left(-\frac{2 J k}{r g(r)}+\frac{3 J k}{2 g^{2}(r)} \frac{\mathrm{d}}{\mathrm{d} r} g(r)\right), \\
& \tilde{A}_{1}^{2}(r)=\frac{-1}{N^{2}(r)} \int \mathrm{d} r\left(-\frac{J}{r^{3}} h(r)+\frac{3 J}{2 r^{2}} \frac{\mathrm{d}}{\mathrm{d} r} h(r)\right) .
\end{aligned}
$$

Note that for $B_{21}^{\prime}(r)=0$, and using the following coordinate transformation:

$$
r^{2}=\ell r^{\prime}, \quad t=\frac{\sqrt{\ell}\left(\varphi^{\prime}-t^{\prime}\right)}{\left(M^{2} \ell^{2}-J^{2}\right)^{\frac{1}{4}}}, \quad \varphi=\frac{r_{+}^{2} t^{\prime}-r_{-}^{2} \varphi^{\prime}}{\sqrt{\ell}\left(M^{2} \ell^{2}-J^{2}\right)^{\frac{1}{4}}},
$$

the dual metric in (32) precisely represents the charged black string solution $[4,5]$. The dual electric and magnetic fields can be obtained in terms of the dual field strengths as follows:

$$
\begin{aligned}
\tilde{E}_{r}^{(a)} & =-\tilde{F}^{01 a}=-\tilde{g}^{00} \tilde{g}^{11} \tilde{F}_{01}^{a}, \quad \tilde{E}_{\varphi}^{(a)}=-\tilde{F}^{02 a} \\
& =-\tilde{g}^{00} \tilde{g}^{21} \tilde{F}_{01}{ }^{a}, \quad \tilde{B}_{z}^{(a)}=r \tilde{F}^{12 a}=-r \tilde{g}^{11} \tilde{g}^{20} \tilde{F}_{01}{ }^{a} \\
& =-\frac{r^{3}}{\ell} \tilde{F}_{01}{ }^{a} .
\end{aligned}
$$

Furthermore, the radial components of three dual electric fields are obtained as

$$
\begin{aligned}
& \tilde{E}_{r}^{(0)}=-\frac{J}{r^{3}} h(r)+\frac{3 J}{2 r^{2}} \frac{d}{d r} h(r), \\
& \tilde{E}_{r}^{(1)}=-\frac{2 J k}{r g(r)}+\frac{3 J k}{2 g^{2}(r)} \frac{d}{d r} g(r), \quad \tilde{E}_{r}^{(2)}=0,
\end{aligned}
$$

and the azimuthal components of them are obtained as follows:

$$
\begin{aligned}
\tilde{E}_{\varphi}^{(0)}= & \left(\frac{\mathrm{d}}{\mathrm{d} r} h(r)-\frac{2 k r}{g(r)}+\frac{k r^{2}}{g^{2}(r)} \frac{\mathrm{d}}{\mathrm{d} r} g(r)\right) \\
& \times\left(-\frac{J}{r^{3}} h(r)+\frac{3 J}{2 r^{2}} \frac{\mathrm{d}}{\mathrm{d} r} h(r)\right), \\
\tilde{E}_{\varphi}^{(1)}= & \left(\frac{\mathrm{d}}{\mathrm{d} r} h(r)-\frac{2 k r}{g(r)}+\frac{k r^{2}}{g^{2}(r)} \frac{\mathrm{d}}{\mathrm{d} r} g(r)\right) \\
& \times\left(-\frac{2 J k}{r g(r)}+\frac{3 J k}{2 g^{2}(r)} \frac{\mathrm{d}}{\mathrm{d} r} g(r)\right), \quad \tilde{E}_{\varphi}^{(2)}=0,
\end{aligned}
$$

and finally the magnetic fields which all are in the $\mathrm{z}$-direction are given by the following relations:

$$
\begin{aligned}
& \tilde{B}_{z}^{(0)}=-\frac{r^{3}}{\ell}\left(-\frac{J}{r^{3}} h(r)+\frac{3 J}{2 r^{2}} \frac{\mathrm{d}}{\mathrm{d} r} h(r)\right), \\
& \tilde{B}_{z}^{(1)}=-\frac{r^{3}}{\ell}\left(-\frac{2 J k}{r g(r)}+\frac{3 J k}{2 g^{2}(r)} \frac{\mathrm{d}}{\mathrm{d} r} g(r)\right), \quad \tilde{B}_{z}^{(2)}=0 .
\end{aligned}
$$

Note that if we select the following forms for arbitrary functions $g(r)$ and $h(r)$ :

$g(r)=\operatorname{Dr} N(r) \mathrm{e}^{-V(r)}, \quad h(r)=\frac{C r}{N(r)} \mathrm{e}^{V(r)}$,

where $D$ and $C$ are arbitrary constants and

$V(r)=\frac{M}{\sqrt{M^{2}+\Lambda J^{2}}} \tanh ^{-1}\left(\frac{M+2 \Lambda r^{2}}{\sqrt{M^{2}+\Lambda J^{2}}}\right)$,

then all magnetic fields in (30) become zero, and therefore, we have only the electric fields in the solution (19), while in the dual solution (32) there are both electric and magnetic fields. This result, as we expect, indicates that here, in string theory, the duality leads to a connection between electric and magnetic fields in the solution (19) and its dual solution (32).

Let us discuss the isometry symmetry along the $t$-direction of the solution (19). One can repeat the above procedure to find the following $t$-dual solution [23] for the solution (19):

$$
\begin{aligned}
\tilde{\mathrm{d} s}^{2}= & \frac{1}{\left(M-\frac{r^{2}}{\ell^{2}}\right)}\left[\mathrm{d} t^{2}+2 \frac{r^{2}}{\ell} \mathrm{d} t \mathrm{~d} \varphi-2 B_{01}^{\prime}(r)\right. \\
& \left(\mathrm{d} t \mathrm{~d} r-\frac{r^{2}}{\ell} d r d \varphi\right)+r^{2}\left(M-\frac{J^{2}}{4 r^{2}}\right) \mathrm{d} \varphi^{2}
\end{aligned}
$$




$$
\begin{gathered}
\left.+\left\{\frac{\left(M-\frac{r^{2}}{\ell^{2}}\right)}{N^{2}(r)}+\left(B_{01}^{\prime}(r)\right)^{2}\right\} \mathrm{d} r^{2}\right], \\
\tilde{B}_{20}(r)=-\frac{J}{2\left(M-\frac{r^{2}}{\ell^{2}}\right)}, \quad \tilde{\phi}(r)=-\frac{1}{2} \ln \left(-M+\frac{r^{2}}{\ell^{2}}\right), \\
\tilde{F}_{21}{ }^{0}(r)=\frac{1}{\left(M-\frac{r^{2}}{\ell^{2}}\right)}\left(\frac{J^{2}}{r^{3}} h(r)+\left(-M+\frac{r^{2}}{\ell^{2}}-\frac{J^{2}}{2 r^{2}}\right) \frac{\mathrm{d}}{\mathrm{d} r} h(r)\right), \\
\tilde{F}_{21}{ }^{1}(r)=\frac{2 k r}{g(r)}+\frac{k r^{2}}{g^{2}(r)\left(M-\frac{r^{2}}{\ell^{2}}\right)}\left(-M+\frac{r^{2}}{\ell^{2}}-\frac{J^{2}}{2 r^{2}}\right) \frac{\mathrm{d}}{\mathrm{d} r} g(r) .
\end{gathered}
$$

For $B_{01}^{\prime}(r)=0$, and using the following coordinate transformation [23]:

$$
\begin{aligned}
r^{2} & =\ell r^{\prime}+M \ell^{2}, \quad t=\frac{r_{+}^{2} \varphi^{\prime}-r_{-}^{2} t^{\prime}}{\ell^{\frac{3}{2}}\left(M^{2} \ell^{2}-J^{2}\right)^{\frac{1}{4}}} \\
\varphi & =\frac{t^{\prime}-\varphi^{\prime}}{\sqrt{\ell}\left(M^{2} \ell^{2}-J^{2}\right)^{\frac{1}{4}}},
\end{aligned}
$$

the $t$-dual metric in (41) precisely reduces to the charged black string solution. Finally, calculations similar to the $\varphi$ dual case show that the $t$-dual solution also leads to a connection between electric and dual magnetic fields for the solution (19) and its $t$-dual solution, respectively.

\section{Conclusions}

We have presented a $2+1$ dimensional low energy string effective action containing gauge fields term which has a gauge symmetry coming from a semi-simple extension of the Poincare (Maxwell) gauge group. The model has led to an extended B-field in the corresponding sigma model. By solving the equations of motion of the string effective action (i.e. the beta function equations), we have obtained two different solutions which both in the sigma model level correspond to the $\mathrm{SL}(2, R)$ WZW models and then both are exact solutions of beta function equations to all orders. Also, it turned out that the two sigma models corresponding to two different solutions are classically canonically equivalent. We have interpreted the gauge field strength tensors related to the Abelian gauge fields solution as electromagnetic field strength tensors and obtained the corresponding electric and magnetic fields. Using a Buscher duality transformation, we have shown that the dual models coincide with the charged black string solution and also have shown that the electric fields of the Abelian solution are related to the magnetic fields of its dual solution.

Acknowledgments We would like to express our gratitude to M.M. Sheikh-Jabbari and F. Darabi for their useful comments. This research was supported by a research fund No. 217D4310 from Azarbaijan Shahid Madani university.

Open Access This article is distributed under the terms of the Creative Commons Attribution 4.0 International License (http://creativecomm ons.org/licenses/by/4.0/), which permits unrestricted use, distribution, and reproduction in any medium, provided you give appropriate credit to the original author(s) and the source, provide a link to the Creative Commons license, and indicate if changes were made. Funded by SCOAP ${ }^{3}$.

Appendix A: S-expansion of the AdS algebra so(2, 2) in $2+1$ dimensions

In this section, we start from the anti-de Sitter algebra $\mathfrak{g}=\operatorname{so}(2,2)$ in $2+1$ dimensions and use the finite abelian semigroup expansion procedure ${ }^{6}$ ( $\mathrm{S}$-expansion) to write both the Maxwell algebra and the semi-simple extension of the Poincaré algebra. ${ }^{7}$ We consider the anti-de Sitter algebra $\mathfrak{g}=\operatorname{so}(2,2)$ with the basis $\bar{X}_{B}=\left\{\bar{P}_{a}, \bar{J}_{a}\right\}$ in $2+1$ dimensions as follows:

$$
\begin{aligned}
& {\left[\bar{J}_{a}, \bar{J}_{b}\right]=\epsilon_{a b c} \bar{J}^{c}, \quad\left[\bar{J}_{a}, \bar{P}_{b}\right]=\epsilon_{a b c} \bar{P}^{c},} \\
& {\left[\bar{P}_{a}, \bar{P}_{b}\right]=\Lambda \epsilon_{a b c} \bar{J}^{c},}
\end{aligned}
$$

where $\Lambda$ is a constant, and $\bar{P}_{a}$ and $\bar{J}_{a}$ are the ordinary translation and Lorentz generators, respectively. We split the AdS algebra $s o(2,2)$ in two subspaces $s o(2,2)=V_{0} \bigoplus V_{1}$, where $V_{0}$ and $V_{1}$ correspond to the Lorentz and translation generators $\bar{J}_{a}$ and $\bar{P}_{a}$, respectively. The subspace structure is such that we have

$\left[V_{0}, V_{0}\right] \subset V_{0}, \quad\left[V_{0}, V_{1}\right] \subset V_{1}, \quad\left[V_{1}, V_{1}\right] \subset V_{0}$.

Now, in the following, we use two different semigroups to expand the AdS algebra by use of the S-expansion procedure, and we obtain both the Maxwell algebra and the semi-simple extension of the Poincaré algebra.

\section{A.1 The Maxwell algebra and the S-expansion} by the semigroup $S$

We first consider the abelian semigroup $S=\left\{\lambda_{0}, \lambda_{1}, \lambda_{2}, \lambda_{3}\right\}$ together with the following multiplication law:

$\lambda_{\alpha} \lambda_{\beta}=\left\{\begin{array}{ll}\lambda_{3} & \text { if }(\alpha+\beta)>2 \\ \lambda_{\alpha+\beta} & \text { if }(\alpha+\beta) \leq 2\end{array}\right.$,

\footnotetext{
6 The full details of the S-expansion procedure have been presented in [18].

7 The Maxwell algebra and the semi-simple extension of the Poincaré algebra in $D$-dimensional spacetime have been obtained by the $\mathrm{S}$ expansion of the $D$-dimensional AdS algebra in $[14,15]$, respectively.
} 
or, equivalently, by the following multiplication table:

\begin{tabular}{l|llll} 
& $\lambda_{0}$ & $\lambda_{1}$ & $\lambda_{2}$ & $\lambda_{3}$ \\
\hline$\lambda_{0}$ & $\lambda_{0}$ & $\lambda_{1}$ & $\lambda_{2}$ & $\lambda_{3}$ \\
$\lambda_{1}$ & $\lambda_{1}$ & $\lambda_{2}$ & $\lambda_{3}$ & $\lambda_{3}$ \\
$\lambda_{2}$ & $\lambda_{2}$ & $\lambda_{3}$ & $\lambda_{3}$ & $\lambda_{3}$ \\
$\lambda_{3}$ & $\lambda_{3}$ & $\lambda_{3}$ & $\lambda_{3}$ & $\lambda_{3}$
\end{tabular}

Note that, for each $\lambda_{\alpha} \in S$, we have $\lambda_{3} \lambda_{\alpha}=\lambda_{3}$, such that $\lambda_{3}$ plays the role of the zero element inside the semigroup $S$ (i.e. $\lambda_{3}=0_{S}$ ). Now, we consider the following subset decomposition $S=S_{0} \bigcup S_{1}$ :

$S_{0}=\left\{\lambda_{0}, \lambda_{2}, \lambda_{3}\right\}, \quad S_{1}=\left\{\lambda_{1}, \lambda_{3}\right\}$,

which is said to be a resonant decomposition; in other words, it is in resonance with the subspace decomposition $\mathfrak{g}=V_{0} \bigoplus V_{1}$ and then satisfies the following resonance condition:

$S_{0} . S_{0} \subset S_{0}, \quad S_{0} . S_{1} \subset S_{1}, \quad S_{1} . S_{1} \subset S_{0}$.

The direct product $S \times \mathfrak{g}$ with basis $\lambda_{\alpha} \bar{X}_{B}$ is a Lie algebra (see Theorem III.1 in [18]). According to Theorem IV.2 in [18], $W_{0} \oplus W_{1}$ is a resonant subalgebra of $S \times \mathfrak{g}$, where we have

$$
\begin{aligned}
W_{0} & =S_{0} \times V_{0}=\left\{\lambda_{0}, \lambda_{2}, \lambda_{3}\right\} \otimes\left\{\bar{J}_{a}\right\} \\
& =\left\{\lambda_{0} \bar{J}_{a}, \lambda_{2} \bar{J}_{a}, \lambda_{3} \bar{J}_{a}\right\} \\
W_{1} & =S_{1} \times V_{1}=\left\{\lambda_{1}, \lambda_{3}\right\} \otimes\left\{\bar{P}_{a}\right\}=\left\{\lambda_{1} \bar{P}_{a}, \lambda_{3} \bar{P}_{a}\right\} .
\end{aligned}
$$

Now, we impose the condition $\lambda_{3} \times \mathfrak{g}=0_{S}$, and remove the whole $0_{S} \times \mathfrak{g}$ sector from the resonant subalgebra. The remaining piece is a Lie algebra and is called the $0_{S}$-reduced algebra (see $0_{S}$-reduction and Theorem VI.1 in [18]). By relabeling the generators as $J_{a} \equiv \lambda_{0} \bar{J}_{a}, k Z_{a} \equiv \lambda_{2} \bar{J}_{a}$, and $\sqrt{\Lambda} P_{a} \equiv \lambda_{1} \bar{P}_{a}$, we obtain the following commutation relations:

$$
\begin{aligned}
& {\left[J_{a}, J_{b}\right]=\lambda_{0} \lambda_{0}\left[\bar{J}_{a}, \bar{J}_{b}\right]=\lambda_{0} \epsilon_{a b c} \bar{J}^{c}=\epsilon_{a b c} J^{c},} \\
& {\left[J_{a}, P_{b}\right]=\frac{1}{\sqrt{\Lambda}} \lambda_{0} \lambda_{1}\left[\bar{J}_{a}, \bar{P}_{b}\right]=\frac{1}{\sqrt{\Lambda}} \lambda_{1} \epsilon_{a b c} \bar{P}^{c}=\epsilon_{a b c} P^{c},} \\
& {\left[P_{a}, P_{b}\right]=\frac{1}{\Lambda} \lambda_{1} \lambda_{1}\left[\bar{P}_{a}, \bar{P}_{b}\right]=\lambda_{2} \epsilon_{a b c} \bar{J}^{c}=k \epsilon_{a b c} Z^{c},} \\
& {\left[J_{a}, Z_{b}\right]=\frac{1}{k} \lambda_{0} \lambda_{2}\left[\bar{J}_{a}, \bar{J}_{b}\right]=\frac{1}{k} \lambda_{2} \epsilon_{a b c} \bar{J}^{c}=\epsilon_{a b c} Z^{c},} \\
& {\left[P_{a}, Z_{b}\right]=\frac{1}{k \sqrt{\Lambda}} \lambda_{1} \lambda_{2}\left[\bar{P}_{a}, \bar{J}_{b}\right]=\frac{1}{k \sqrt{\Lambda}} \epsilon_{a b c} \lambda_{3} \bar{P}^{c}=0,} \\
& {\left[Z_{a}, Z_{b}\right]=\frac{1}{k^{2}} \lambda_{2} \lambda_{2}\left[\bar{J}_{a}, \bar{J}_{b}\right]=\frac{1}{k^{2}} \epsilon_{a b c} \lambda_{3} \bar{J}^{c}=0,}
\end{aligned}
$$

where we have used the commutation relations of the AdS algebra (43) and the multiplication law (46) of the semigroup $S$. The obtained algebra (51) coincides with the Maxwell algebra $[9,10]$.

A.2 The semi-simple extension of the Poincaré algebra and the S-expansion by the semigroup $\bar{S}$

We consider the abelian semigroup $\bar{S}=\left\{\bar{\lambda}_{0}, \bar{\lambda}_{1}, \bar{\lambda}_{2}\right\}$ together with the following multiplication law:

$\bar{\lambda}_{\alpha} \bar{\lambda}_{\beta}=\left\{\begin{array}{ll}\bar{\lambda}_{\alpha+\beta-2} & \text { if }(\alpha+\beta)>2 \\ \bar{\lambda}_{\alpha+\beta} & \text { if }(\alpha+\beta) \leq 2\end{array}\right.$,

or, equivalently, by the following multiplication table:

\begin{tabular}{l|lll} 
& $\bar{\lambda}_{0}$ & $\bar{\lambda}_{1}$ & $\bar{\lambda}_{2}$ \\
\hline $\bar{\lambda}_{0}$ & $\bar{\lambda}_{0}$ & $\bar{\lambda}_{1}$ & $\bar{\lambda}_{2}$ \\
$\bar{\lambda}_{1}$ & $\bar{\lambda}_{1}$ & $\bar{\lambda}_{2}$ & $\bar{\lambda}_{1}$ \\
$\bar{\lambda}_{2}$ & $\bar{\lambda}_{2}$ & $\bar{\lambda}_{1}$ & $\bar{\lambda}_{2}$
\end{tabular}

Now, we consider the following subset decomposition $\bar{S}=$ $\bar{S}_{0} \bigcup \bar{S}_{1}$ :

$\bar{S}_{0}=\left\{\bar{\lambda}_{0}, \bar{\lambda}_{2}\right\}, \quad \bar{S}_{1}=\left\{\bar{\lambda}_{1}\right\}$

which is in resonance with the subspace decomposition $\mathfrak{g}=V_{0} \bigoplus V_{1}$ (resonant decomposition) and then satisfies the following resonance condition:

$\bar{S}_{0} \cdot \bar{S}_{0} \subset \bar{S}_{0}, \quad \bar{S}_{0} \cdot \bar{S}_{1} \subset \bar{S}_{1}, \quad \bar{S}_{1} \cdot \bar{S}_{1} \subset \bar{S}_{0}$.

The direct product $\bar{S} \times \mathfrak{g}$ with basis $\bar{\lambda}_{\alpha} \bar{X}_{B}$ is a Lie algebra (see Theorem III.1 in [18]). According to Theorem IV.2 in [18], $\bar{W}_{0} \oplus \bar{W}_{1}$ is a resonant subalgebra of $\bar{S} \times \mathfrak{g}$ where we have

$$
\begin{aligned}
& \bar{W}_{0}=\bar{S}_{0} \times V_{0}=\left\{\bar{\lambda}_{0}, \bar{\lambda}_{2}\right\} \otimes\left\{\bar{J}_{a}\right\}=\left\{\bar{\lambda}_{0} \bar{J}_{a}, \bar{\lambda}_{2} \bar{J}_{a}\right\}, \\
& \bar{W}_{1}=\bar{S}_{1} \times V_{1}=\left\{\bar{\lambda}_{1}\right\} \otimes\left\{\bar{P}_{a}\right\}=\left\{\bar{\lambda}_{1} \bar{P}_{a}\right\} .
\end{aligned}
$$

Relabeling the generators as $J_{a} \equiv \bar{\lambda}_{0} \bar{J}_{a}, \quad-\frac{k}{\lambda} Z_{a} \equiv \bar{\lambda}_{2} \bar{J}_{a}$ and $\sqrt{-\frac{\Lambda}{\lambda}} P_{a} \equiv \bar{\lambda}_{1} \bar{P}_{a}$, we obtain the following commutation relations:

$$
\begin{aligned}
{\left[J_{a}, J_{b}\right] } & =\bar{\lambda}_{0} \bar{\lambda}_{0}\left[\bar{J}_{a}, \bar{J}_{b}\right]=\bar{\lambda}_{0} \epsilon_{a b c} \bar{J}^{c}=\epsilon_{a b c} J^{c}, \\
{\left[J_{a}, P_{b}\right] } & =\sqrt{-\frac{\lambda}{\Lambda}} \bar{\lambda}_{0} \bar{\lambda}_{1}\left[\bar{J}_{a}, \bar{P}_{b}\right] \\
& =\sqrt{-\frac{\lambda}{\Lambda}} \bar{\lambda}_{1} \epsilon_{a b c} \bar{P}^{c}=\epsilon_{a b c} P^{c}, \\
{\left[P_{a}, P_{b}\right] } & =-\frac{\lambda}{\Lambda} \bar{\lambda}_{1} \bar{\lambda}_{1}\left[\bar{P}_{a}, \bar{P}_{b}\right]=-\lambda \bar{\lambda}_{2} \epsilon_{a b c} \bar{J}^{c}=k \epsilon_{a b c} Z^{c}, \\
{\left[J_{a}, Z_{b}\right] } & =-\frac{\lambda}{k} \bar{\lambda}_{0} \bar{\lambda}_{2}\left[\bar{J}_{a}, \bar{J}_{b}\right]=-\frac{\lambda}{k} \bar{\lambda}_{2} \epsilon_{a b c} \bar{J}^{c}=\epsilon_{a b c} Z^{c}, \\
{\left[P_{a}, Z_{b}\right] } & =-\frac{\lambda}{k} \sqrt{-\frac{\lambda}{\Lambda}} \bar{\lambda}_{1} \bar{\lambda}_{2}\left[\bar{P}_{a}, \bar{J}_{b}\right] \\
& =-\frac{\lambda}{k} \sqrt{-\frac{\lambda}{\Lambda}} \bar{\lambda}_{1} \epsilon_{a b c} \bar{P}^{c}=-\frac{\lambda}{k} \epsilon_{a b c} \bar{P}^{c}, \\
{\left[Z_{a}, Z_{b}\right] } & =\frac{\lambda^{2}}{k^{2}} \bar{\lambda}_{2} \bar{\lambda}_{2}\left[\bar{J}_{a}, \bar{J}_{b}\right]=\frac{\lambda^{2}}{k^{2}} \bar{\lambda}_{2} \epsilon_{a b c} \bar{J}^{c}=-\frac{\lambda}{k} \epsilon_{a b c} \bar{Z}^{c},
\end{aligned}
$$


where we have used the commutation relations of the AdS algebra (43) and the multiplication law (53) of the semigroup $\bar{S}$. The obtained algebra (57) matches the semi-simple extension of the Poincaré algebra [12].

\section{References}

1. M. Banados, C. Teitelboim, J. Zanelli, The black hole in three dimensional space time. Phys. Rev. Lett. 69, 1849 (1992). arXiv:hep-th/9204099

2. M. Banados, M. Henneaux, C. Teitelboim, J. Zanelli, Geometry of the 2+1 black hole. Phys. Rev. D 48, 1506 (1993). arXiv:gr-qc/9302012

3. E. Witten, On string theory and black holes. Phys. Rev. D 44, 314 (1991)

4. J.H. Horne, G.T. Horowitz, Exact black string solutions in three dimensions. Nucl. Phys. B 368, 444-462 (1992). arXiv:hep-th/9108001

5. G.T. Horowitz, D.L. Welch, Exact three dimensional black holes in string theory. Phys. Rev. Lett. 71, 328-331 (1993). arXiv:hep-th/9302126

6. J. Maldacena, H. Ooguri, Strings in AdS3 and SL(2, R) WZW model. Part 1: the spectrum. J. Math. Phys. 42, 2929 (2001, Special issue on M Theory). arXiv:hep-th/0001053

7. J. Maldacena, H. Ooguri, J. Son, Strings in AdS3 and the SL(2, R)WZW model. Part 2: Euclidean black hole. J. Math. Phys. 42, 2961 (2001, Special issue on M Theory). arXiv:hep-th/0005183

8. J.M. Maldacena, H. Ooguri, Strings in $\operatorname{AdS}(3)$ and the $\operatorname{SL}(2, R)$ WZW model. Part 3. Correlation functions. Phys. Rev. D 65, 106006 (2002). arXiv:hep-th/0111180

9. H. Bacry, P. Combe, J.L. Richard, Group-theoretical analysis of elementary particles in an external electromagnetic field. 1. The relativistic particle in a constant and uniform field. Nuovo Cim. A 67, 267-299 (1970). ibid. A 70, 289-312 (1970)

10. R. Schrader, The Maxwell group and the quantum theory of particles in classical homogeneous electromagnetic fields. Fortsch. Phys. 20, 701-734 (1972)
11. J.A. de Azcarraga, K. Kamimura, J. Lukierski, Generalized cosmological term from Maxwell symmetries. Phys. Rev. D 83, 124036 (2011). arXiv:1012.4402 [hep-th]

12. D.V. Soroka, V.A. Soroka, Gauge semi-simple extension of the Poincaré group. Phys. Lett. B 707, 160-162 (2012). arXiv:1101.1591 [hep-th]

13. S. Hoseinzadeh, A. Rezaei-Aghdam, $2+1$ dimensional gravity from Maxwell and semi-simple extension of the Poincare gauge symmetric models. Phys. Rev. D 90, 084008 (2014). arXiv: 1402.0320 [hep-th]

14. P. Salgado, R.J. Szabo, O. Valdivia, Topological gravity and transgression holography. Phys. Rev. D 89, 084077 (2014). arXiv: 1401.3653 [hep-th]

15. J. Díaz, O. Fierro, F. Izaurieta, N. Merino, E. Rodrguez, P. Salgado, O. Valdivia, A generalized action for (2+1)-dimensional ChernSimons gravity. J. Phys. A 45, 255207 (2012). arXiv:1311.2215 [hep-th]

16. E.S. Fradkin, A.A. Tseytlin, Quantum string theory effective action. Nucl. Phys. B 261, 1-27 (1985)

17. C.G. Callan, E.J. Martinec, M.J. Perry, D. Friedan, Strings in background fields. Nucl. Phys. B 262, 593 (1985)

18. F. Izaurieta, E. Rodríguez, P. Salgado, Expanding Lie (super)algebras through abelian semigroups. J. Math. Phys. 47, 123512 (2006). arXiv:hep-th/0606215

19. F. Izaurieta, A. Perez, E. Rodrguez, P. Salgado, Dual formulation of the Lie algebra S-expansion procedure. J. Math. Phys. 50, 073511 (2009). arXiv:0903.4712 [hep-th]

20. K. Sfetsos, Canonical equivalence of non-isometric $\sigma$ - models and PoissonLie T-duality. Nucl. Phys. B 517, 549-566 (1998). arXiv:hep-th/9710163

21. T.H. Buscher, Path integral derivation of quantum duality in nonlinear sigma models. Phys. Lett. B 201, 466 (1988)

22. T.H. Buscher, A symmetry of the string background field equations. Phys. Lett. B 194, 59 (1987)

23. D.L. Welch, Timelike duality. Phys. Rev. D 50, 6404 (1994). arXiv:hep-th/9405070 